\title{
Mechanistic understanding of electron-beam induced akaganeite nanorod dissolution
}

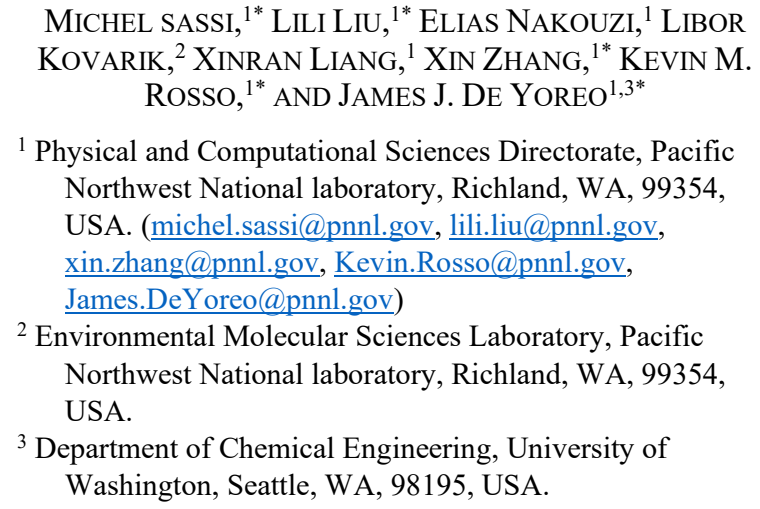

${ }^{1}$ Physical and Computational Sciences Directorate, Pacific Northwest National laboratory, Richland, WA, 99354, USA. (michel.sassi@pnnl.gov, lili.liu@pnnl.gov, xin.zhang@pnnl.gov, Kevin.Rosso@pnnl.gov, James.DeYoreo@pnnl.gov)

${ }^{2}$ Environmental Molecular Sciences Laboratory, Pacific Northwest National laboratory, Richland, WA, 99354, USA.

${ }^{3}$ Department of Chemical Engineering, University of Washington, Seattle, WA, 98195, USA.

Dissolution of redox-active metal oxides plays a key role in technological applications and environmental settings. Despite its widespread significance, mechanisms of metal-oxide dissolution remain poorly understood at the atomic level. A combination of in-situ liquid phase transmission electron microscopy (LTEM) and deterministic simulations is used to explore the dissolution behaviour of akaganeite nanorods under electron beam, both with and without the presence of buffer. In pure water, akageneite dissolves along both [001] and [010] directions, however, the dissolution behaviour changes depending on the concentration and type of buffer present in the solution. With $100 \mathrm{mM}$ of bistris, the dissolution was effectively inhibited while with tris- $\mathrm{HCl}$ only a dissolution along the [001] direction is observed, even at low buffer concentration. Deterministic simulations suggest that superoxide, aqueous electrons and protons play a role in akaganeite dissolution, and that bistris effectively reduces the availability of superoxide and aqueous electrons, two major contributors, therefore delaying dissolution. This study shows that water radiolysis contributes to the reduction of $\mathrm{Fe}(\mathrm{III})$ to release $\mathrm{Fe}(\mathrm{II})$, thus promoting dissolution of iron oxyhydroxides. 